\title{
Implementation of 3D Graphics and Image Algorithm Using Matlab
}

\author{
Wan-Bo Yu, Yu Huang \\ Department of Computer Science, College of Information, Dalian University, Dalian, China
}

\section{Email address:}

yuwanbo@dlu.edu.cn (Wan- Bo Yu)

\section{To cite this article:}

Wan-Bo Yu, Yu Huang. Implementation of 3D Graphics and Image Algorithm Using Matlab. Science Discovery.

Vol. 5, No. 6, 2017, pp. 416-419. doi: 10.11648/j.sd.20170506.13

Received: August 6, 2017; Accepted: September 8, 2017; Published: October 28, 2017

\begin{abstract}
Computer graphics and image processing are hot topics in the academic and engineering fields. There are many algorithms for three-dimensional graphics rendering and image processing which using $\mathrm{C}$ language or JAVA language is difficult to achieve. Therefore, the use of MATLAB to achieve three-dimensional graphics rendering algorithms and image processing algorithms. Some examples are given to illustrate and discuss some research work. For some experimental work, MATLAB has many advantages, and it is a better experimental tool platform.
\end{abstract}

Keywords: Computer Graphics, Image Processing, Matlab

\section{三维图形及图像算法的MATLAB实现}

于万波, 黄昱

信息工程学院计算机系, 大连大学, 大连, 中国

邮箱

yuwanbo@dlu.edu.cn（于万波）

摘要：计算机图形绘制与图像处理是目前学术界与工程领域研究的热点。计算机三维图形绘制以及图像处理中有很多 算法用C语言或者Java语言实现起来比较困难, 所以研究使用Matlab实现三维图形绘制算法以及图像处理算法。给出一 些实例进行相应的说明, 结合一些研究工作分析讨论。对于一些实验性工作, Matlab有很多优点, 是一个较好的实验 工具平台。

关键词：计算机图形学, 图像处理, Matlab

\section{1. 引言}

专门用来进行绘图及图像处理的软件有很多, 例如, Photoshop, Coreldraw, AutoCAD等。另外, 还有一些软件, 如Flash、3D-max、Maya等, 除了能够完成绘图与建模外, 还提供了强大的动画制作功能。除了这些专用软件, 诸多 的语言也都提供了画图语句, 提供了用来制作动画的函 数。在众多的软件与语言中, 选择哪种语言或者软件来讲
或研究计算机图形绘制与图像处理是一个关键的问题 [1-6]。

其实, 语言绘图与软件绘图之间并没有截然的区别, 只是对于非计算机研究人员, 使用软件绘图更加方便而 已。在有些时候进行软件开发与工程实验时, 例如绘制图 形动画、进行图像处理时最好用语言来实现。Matlab是一 
个集图形动画制作语言、软件于一体的工具, 使用Matlab 是一个比较好的选择[7-10]。目前很多研究生的论文是基 于Matlab实现的 [11-13]。

选择Matlab语言, 因为这种语言提供了三维绘制函 数, 而且是基于矩阵的。Matlab在界面上 (和语言中) 提 供了变换视点等功能, 可以直观的从各个角度观察一个绘 制好的三维物体。Matlab也提供了四维数组函数用来存咜 序列图像, 这个功能非常有利于处理三维图形学中序列图 像的一些算法[14-16]。长期以来, 三维图形学教学中, 算 法实现是一个难点, 例如, 不论是用 $\mathrm{C}$ 语言还是用 $\mathrm{VB}$, 都 由于三维算法的复杂性而不易被学生接受; 而选择 OPENGL来处理三维问题, 又由于其函数的复杂程度过高 而隐藏了三维图形的绘制原理, 所以, 可以尝试选用 Matlab作为工具来实现三维算法。

另外, Matlab中, 有虚拟现实工具箱, 其中的V-Realm Builde软件能够很容易实现简单的三维造型、动画制作以 及虚拟现实效果, 完全能够满足教学的需要。在这个意义 上, Matlab既提供了绘图语言, 也提供了绘图软件, 不需 要再使用其他软件支持。

可以在教学中讲解如何用Matlab语言来模拟实现绘 图软件的功能, 例如实现Flash中的动画制作功能等等 [17]。加入这些内容, 一方面让学生加深对绘图原理的理 解, 一方面通过这种方法学习有关的经典计算机图形学的 内容。目的是一方面使学生掌握基本的图形动画生成原理 及算法, 对各个层次的绘图有个贯通的理解; 另一方面让 学生掌握一些实用的图形生成技术。这种讲授方法可以用 在于计算机专业或一些图形学相关专业学生, 也可以用于 专门学习动漫专业的学生。如果是动漫专业的学生, 建议 除了这个课程外, 还应学习两类课程, 一个是绘图软件及 语言的熟练使用, 一个是作品创意之类的实践课程。因为 基于MATLAB的各种教学更适合于介于语言使用与创作 作品之间 [18], 是适合于用来讲解原理的。

\section{2. 三维绘制算法的MATLAB实现}

下面通过几个例子说明Matlab在实现三维绘制方面 具有极大的优势。

例如, 使用Matlab, 构造下面很短的程序就可以绘制 出贝塞尔曲面等。

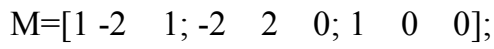

$\mathrm{B}(:,:, 1)=\operatorname{rand}(3,3) ; \mathrm{B}(:,:, 2)=\operatorname{rand}(3,3) ; \mathrm{B}(:,:, 3)=\operatorname{rand}(3,3)$;

for $\mathrm{i}=1: 3$

$\mathrm{Q}(:,, ; \mathrm{i})=\mathrm{M} * \mathrm{~B}(:,, ; \mathrm{i}) * \mathrm{M}^{\prime}$;

for $\mathrm{u}=0: 1$

for $\mathrm{w}=0: 1$

$\mathrm{u} 1=\mathrm{u}^{\wedge} 2 ; \mathrm{w} 1=\mathrm{w}^{\wedge} 2 ;$

$\mathrm{S}(\mathrm{u}+1, \mathrm{w}+1, \mathrm{i})=\left[\begin{array}{lll}\mathrm{u} 1 & \mathrm{u} & 1\end{array}\right]^{*} \mathrm{Q}(:,:, \mathrm{i}) *\left[\begin{array}{lll}\mathrm{w} 1 & \mathrm{w} & 1\end{array}\right] ;$ end

end

end

plot3(B(:,:,1),B(:,:,2),B(:,:,3),'.')

hold on

$\operatorname{surf}(\mathrm{S}(:,:, 1), \mathrm{S}(:,:, 2), \mathrm{S}(:,:, 3))$
该程序绘制出的双三次贝塞尔曲面如图1所示。该例 控制点矩阵 $\mathrm{B}$ 是随机生成的, 可以根据需要给 $\mathrm{B}$ 赋值。控 制点也用“点”绘制出来, 以便观察。

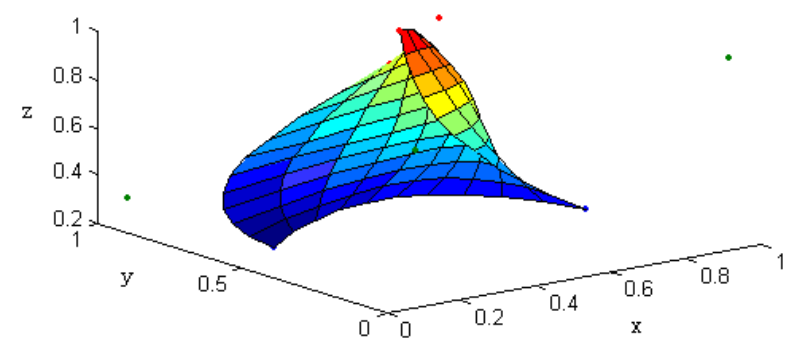

图1 双三次贝塞尔曲面。

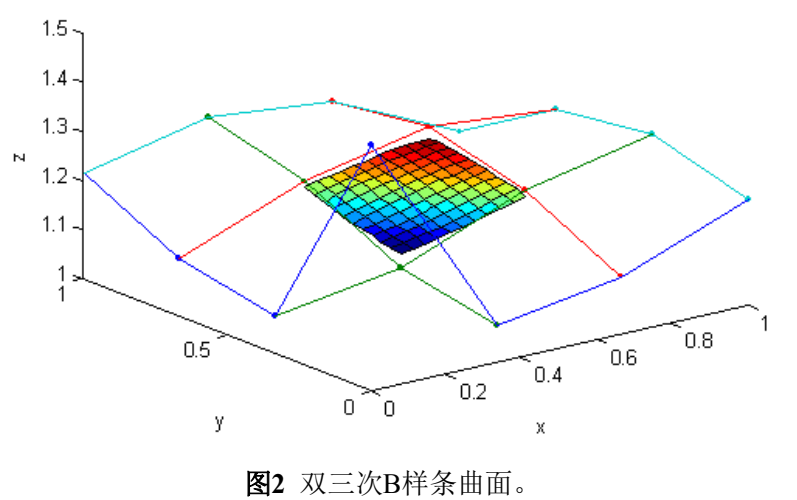

同样, 很容易绘制出带网格的双三次B样条曲面, 如 图2所示。

上面绘图程序比较简单, 代码少, 图形真实, 易于修 改。能够很容易调整控制点观察图形的变化。

另外, MATLAB还提供了样条函数工具箱, 其中有几 十个样条函数, 可以完成各种插值拟合曲线曲面的绘制。

再如, 使用Matlab可以很容易绘制出图 3 与图4所示的 效果图。绘制图 3, 只需要 $\mathrm{Z}=\mathrm{ones}(2,1) ; \mathrm{h}=\mathrm{bar} 3(\mathrm{Z})$; $\operatorname{set}(\mathrm{h}(1)$,'FaceAlpha',0.2);axis off这几个语句。

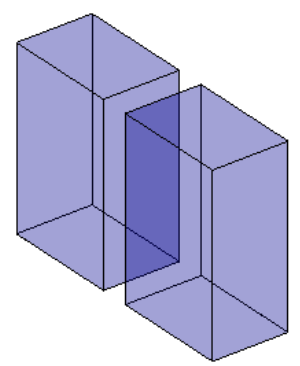

图3 透明效果。
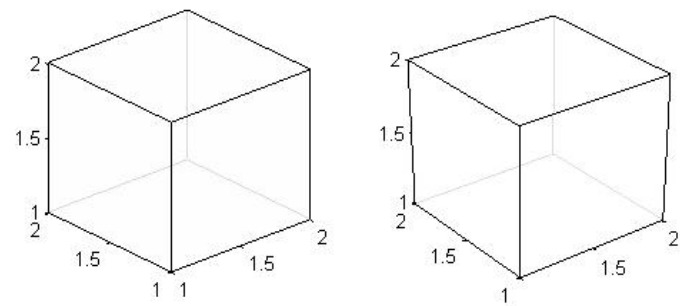

图4 两种投影方式。 
图形学教学中, 隐藏面程序设计是个难点。关于隐藏 面的检测, 在这里, 首先使用语句 sphere(5) 绘制出图5。 然后，使用程序段

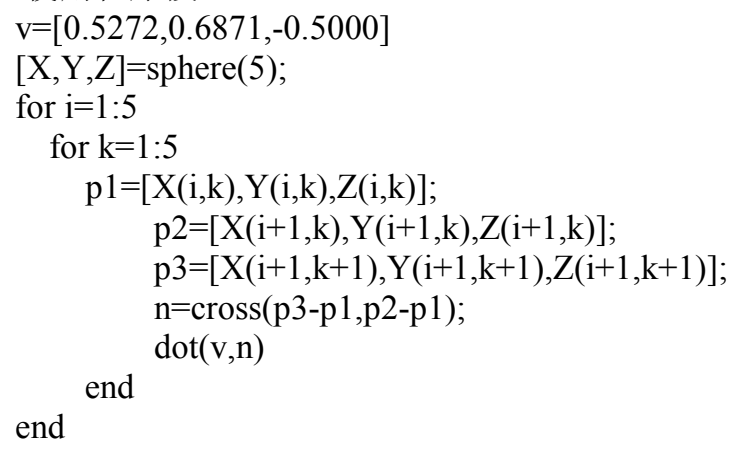

计算出各个面法向量与视向量的内积, 根据内积就可 以判断各个面的可见性了。

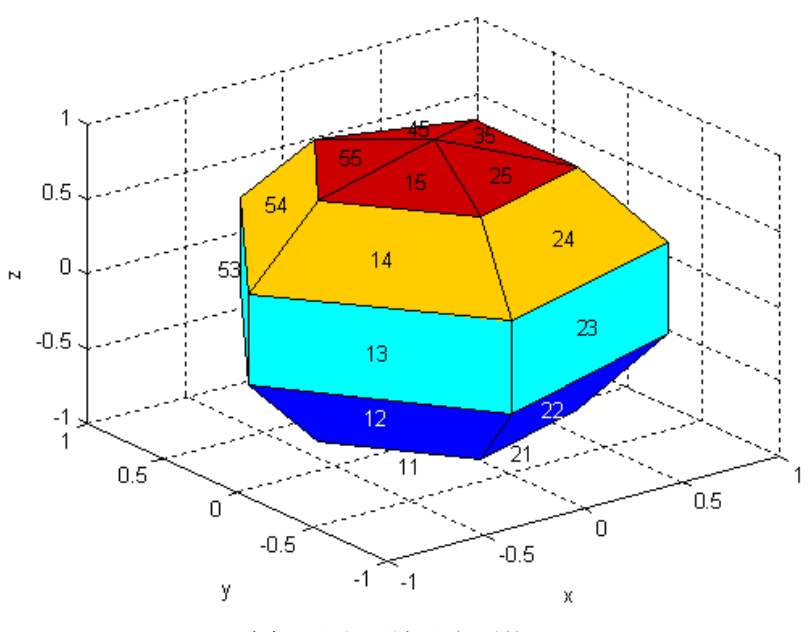

图5 无光照效果多面体。

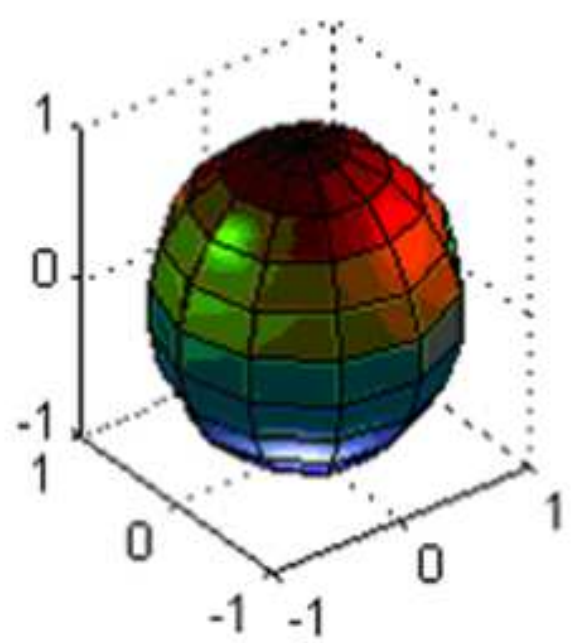

图6 有光照效果的球。

光照的计算程序也很简单。根据面的可见性, 比较容 易绘制出图6所示复杂光照图形。而这些工作在使用一些 语言实现时都是异常复杂。

在安装 Matlab 的时候, 只要选择 Virtual Reality Toolbox复选框, 就可以把虚拟现实工具箱安装在机器上。
V-Realm Builder是三维建模软件, 使用它一方面可以 减少使用VRML编程的工作量, 一方面可以直接观察三维 效果。V-Realm Builder与其他三维绘图软件的使用方法基 本相同, 可以使用该软件进行虚拟现实教学。

\section{MATLAB图像处理}

使用 $\mathrm{VC}++$ 等读取jpg 等格式图像比较复杂, 而在 Matlab中, 一个语句就可以读出jpg, BMP, tif等多种格式的 图像。例如下面语句段, 读出并显示图像如图7所示:

for $\mathrm{i}=1: 10$

$\mathrm{k}=\mathrm{int} 2 \mathrm{str}(\mathrm{i})$;

$\mathrm{k} 1=\operatorname{strcat}\left(' \mathrm{~F}:\left\lfloor\ldots . . . I^{\prime}, \mathrm{k}\right.\right.$, ,.pgm');

$\mathrm{H}(:, ;, \mathrm{i})=\mathrm{imread}(\mathrm{k} 1)$;

$\mathrm{A}(:,:)=\mathrm{H}(:,:, \mathrm{i})$;

$\operatorname{subplot}(2,5, \mathrm{i})$

imshow (A)

end
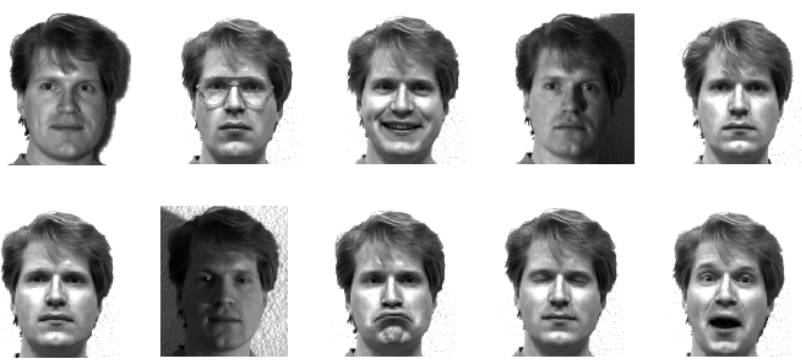

图7 使用matlab显示的人脸图像。

在进行图像识别时, 可以借助于Matlab其他工具箱中 的函数进行程序设计, 例如可以直接利用自相关函数等实 现简单的识别, 利用几何特点识别数字汉字等。例如使用 下面程序就可以识别出图 8 中的数字 8 与数字 2 。

$A=$ imread('D: Ishibie8san.bmp');

$\mathrm{L}=$ bwlabel $(\mathrm{A})$;

$\mathrm{e}=$ imfeature(L,'all');

imshow(A)

for $i=1: 19$

if e(i).EulerNumber $==-1$

$\operatorname{disp}($ '第'\&i\&'个区域是 8 ')

end

if $\mathrm{e}(\mathrm{i})$.EulerNumber $==1 \quad \& \quad \mathrm{e}(\mathrm{i})$.Eccentricity $>0.8 \quad \&$

e(i). Eccentricity $<0.9$

$\operatorname{disp}($ '第'\&i\&'个区域是2')

end

end

\section{1}

图8 待识别的数字。

文献[6]中的识别程序少于 100 行, 完成了复杂的识别 任务, 并且识别准确率高达百分之九十多。 


\section{4. 结论}

与OpenGL以及OpenVC等相比, 在许多场合下Matlab 具有明显的优势。特别是数学、信息科学、电子等相关专 业的本科生与研究生, 适合于选择Matlab作为图形图像的 教学与科研工具。方案的制定对于整个课程、整个项目的 研究起到至关重要的作用。一种工具的选择是一门课程教 学、一个项目研究方案制定的一个内容, 根据具体问题可 以选择使用合适的工具。

该文章属于辽宁省教育科学“十三五”规划立项课题 《归纳为主导的层嵌式立体教学模式研究》(JG17DB037) 的研究结果。

\section{参考文献:}

[1] 拓万兵, 吴凤民.利用MATLAB实现DEM可视化方法研究 [J]. 测绘地理信息, 2017,42(04):32-34。

[2] 田学飞, 利用Matlab解决高等数学中的三维图形问题 [J]. 数 理化解题研究, 2017,(06):2。

[3] 间秋孟, 林超, 高佳, 于尧, 李昕亚, 董默. 基于MATLAB 的医学图像处理系统设计与应用 $[\mathrm{J}]$. 软件, 2017,38(02): 51-55。

[4] 杨保华, 王菁, 梁欣. Matlab在《数字图像处理》教学中 的应用研究 $[J]$. 教育教学论坛, 2016,(02): 171-172。

[5] 杜云明, 王全, 徐建东.基于Matlab的“数字图像处理”教学 [J].电气电子教学学报, 2015,37(04):111-114。

[6] 于万波, 王大庆.曲面迭代的混沌特性及其在人脸识别中的 应用, 计算机辅助设计与图形学学报 [J], 27(12): 2264-2271, 2015。
[7] 周宇, 杜兰, 王鹏辉, 姚若玉.MATLAB课程教学实践及图 像处理应用 $[J]$. 高校实验室工作研究, 2017,(02):1-3。

[8] 凌秀华, 卢文彪, 王耐, 梁丽金, 李熙灿.基于图像处理技 术的麦冬药材特征提取与识别 [J]. 辽宁中医杂志, 2017, 44 (07): 1460-1462。

[9] 毕美华, 杨国伟, 周雪芳, 曾然, 胡丞, 李齐良.MATLAB 寓教于乐教学模式的探讨 [J]. 高教学刊, 2017,(11): $86-87+90$ 。

[10] 姚尧, 胡淑金, 李红亮, 孙银合, 常子栋.基于Matlab的医 学影像图像处理设计 $[\mathrm{J}]$. 数字技术与应用, 2017, (02): 167-169。

[11] 王健. 基于Matlab的车牌识别系统[D]. 吉林大学, 2015。

[12] 潘运平. 基于 web数据挖掘的智能导购系统设计与实现 [D]. 电子科技大学, 2015。

[13] 韩光. 基于视频图像处理的公交车内拥挤检测算法研究 [D]. 长安大学, 2016。

[14] 陈小改. 图像处理技术中matlab方面的应用 $[\mathrm{J}]$. 自动化与 仪器仪表, 2017, (06): 214-215。

[15] 宗凯, 王星.指纹图像特征点提取算法研究[J]. 信息技术, 2017, (05): 147-149+152。

[16] 杨洁, 梅向辉, OLAF Hellwich.基于MATLAB的图像采集 系统研究——在基于视觉的智能监控系统中 $[\mathrm{J} / \mathrm{OL}]$. 南京 邮电大学学报(自然科学版), 2016, 36 (06): 96-99+104。

[17] 谭延峥, 李舜酩, 江星星. 复杂曲线图像的数据提取方法 [J]. 电子测量技术，2016, 39 (12): 159-163。

[18] 赵增顺, 郭为民, 刘伟健, 单蓉, 陈盼飞, 肖树一, 滕升 华, 孙农亮.Matlab图像处理的双语实践与思考 $[\mathrm{J}]$. 实验室科 学, 2016, 19 (04): 97-99。 